\title{
Importance of Performing Corneal Topography Before Cataract Surgery
}

\author{
Jennifer Loh, MD \\ Ophthalmologist, South Florida Vision Associates, Fort Lauderdale, Florida, US
}

\begin{abstract}
Corneal topography assessment has become increasingly important for surgical planning in patients scheduled for cataract surgery. One of the main reasons is the increased expectations for reduced spectacle dependence following cataract surgery, whether patients are selecting an advanced technology intraocular lens (ATIOL), such as a toric or multifocal IOL, or even when choosing a monofocal IOL. Preoperative topography enables surgeons to determine whether a patient is an appropriate candidate for an ATIOL, and also enables surgeons to identify corneal conditions that are contraindications to certain types of IOLS, especially as many corneal conditions are not visible on slit lamp exam. This article will focus on the value provided by preoperative corneal topography prior to cataract surgery and discuss clinical situations where it has been critical in guiding IOL selection.
\end{abstract}

\section{Keywords}

Corneal topography, intraocular lens, preoperative topography

Disclosure: Jennifer Loh, MD, has no conflicts of interest to declare. No funding was received in the publication of this article.

Open Access: This article is published under the Creative Commons Attribution Noncommercial License, which permits any noncommercial use, distribution, adaptation, and reproduction provided the original author(s) and source are given appropriate credit.

Received: August 10, 2015 Accepted: September 14, 2015 Citation: US Ophthalmic Review, 2015;8(2):92-5 DOI: http://dol.org/10.17925/USOR.2015.08.02.92

Correspondence: Jennifer Loh, MD, South Florida Vision Associates, 2900 Cypress Creek Road, Suite \#1, Fort Lauderdale, FL 33309, US. E: JenniferLohMD@gmail.com

With the increasing use of advanced technology intraocular lenses (ATIOLS), the need for careful preoperative assessment is critical for both determination of a patient's candidacy for an ATIOL and the identification of patients who are not appropriate candidates. ${ }^{1}$ Currently, topography is not considered part of the official standard preoperative workup for cataract surgery patients and is not reimbursed by insurances in the US when performed without a known corneal condition. ${ }^{2}$ Unfortunately for cataract surgery patients, there are a number of corneal pathologies that cannot be identified with keratometry, biometry, and slit lamp exam, which are considered the standard, insurance-covered preoperative tests. With corneal topography, critical information on corneal shape can be obtained. Conditions such as irregular corneal astigmatism, early or moderate keratoconus ( $\mathrm{KCN}$ ), and pellucid marginal degeneration can be identified. ${ }^{3}$ Placido disc corneal topography can also provide details on the status of the ocular surface. In particular, common ocular surface conditions, which can be virtually invisible on slit lamp exam, such as ocular surface disease or epithelial basement corneal dystrophy, can be identified. ${ }^{4}$ Treating these ocular surface conditions prior to proceeding with cataract surgery can improve visual outcomes. In addition, corneal topography is especially important in patients with a history of previous corneal refractive surgery, as topography will show whether a patient underwent previous myopic or hyperopic corneal laser refractive surgery, and will show whether the optical zone is centered or decentered..$^{5}$ Topography also aids surgeons in the decision as to whether a patient is an appropriate candidate for an ATIOL. For instance, patients with irregular or skewed astigmatism are often not good candidates for presbyopic IOLs, and may also not be appropriate candidates for a toric IOL. Overall, corneal topography can provide essential information for patients scheduled for cataract surgery and surgeons should consider performing corneal topography for all patients prior to surgery. ${ }^{6}$

\section{Dry Eye/Blepharitis/Ocular Surface Disease}

Ocular surface disease, including dry eye and blepharitis, are common in patients scheduled for cataract surgery. The Prospective Health Assessment of Cataract Patients Ocular surface (PHACO) Study evaluated the incidence of dry eye prior to cataract surgery, ${ }^{7}$ and was conducted at nine centers across the US, and surprisingly found that most patients scheduled for cataract surgery did not report the typical dry eye symptoms, such as ocular irritation of foreign body sensation. However, more than $75 \%$ of patients had signs consistent with dry eye. Of particular concern, more than $62.9 \%$ had a tear break-up time (TBUT) of less than 5 seconds. Rapid TBUT is of great concern, as automated keratometry with the LENSTAR ${ }^{\circledR}$ (Haag Streit, Koeniz, Switzerland) and IOLMaster ${ }^{\circledR}$ (Carl Zeiss Meditec AG, Jena, Germany), expect that the tear film will be stable when the tests are conducted. A rapid TBUT will lead to inaccurate keratometry readings, which will impact the IOL calculations, and can result in a poor visual outcome. 8,9 of similar concern is blepharitis/ meibomian gland disease (MGD). ${ }^{8}$ A study evaluating the incidence of blepharitis in patients scheduled for cataract surgery found that $59 \%$ of patients had this condition. ${ }^{10}$ As it is also associated with a rapid TBUT, identifying and treating MGD can potentially make a difference in surgical planning and visual outcomes. 
While most patients may not be aware preoperatively that they have symptoms of ocular irritation, dry eye can lead to reported postoperative symptoms of ocular irritation, refractive surprises based upon IOL miscalculations, and even cataract surgery being inappropriately performed on patients whose symptomatic visual complaints are more due to dry eye than actual cataract.? If patients are not aware that they have dry eye preoperatively, then they will likely attribute the cataract surgery as the cause of their dry eye. An abnormal ocular surface can lead to inaccurate keratometry that will result in inaccurate IOL calculations., ${ }^{9,11}$ In addition to the wrong spherical power selection, an error in magnitude of axis and magnitude of cylinder power can occur when selecting a toric IOL or planning for a limbal relaxing incision (LRI). With these concerns in mind, it is important to effectively treat dry eye prior to the final preoperative testing. ${ }^{8}$ For example, in Figure 1, a patient underwent IOLMaster, which suggested that a 20.0 diopter lens should have yielded the desired refractive outcome. However, upon closer examination of the associated topography, dry eye can be diagnosed based upon the multiple areas of irregularity.

Upon treatment of the patient's dry eye with topical cyclosporine, a repeat topography and optical biometry was performed. As can be seen in Figure 2, the previously selected 20.0 diopter lens would have resulted in a hyperopic surprise.

While corneal topography can reveal patients who have ocular surface disease, one can also review the IOLMaster, which takes three keratometry readings for each eye. The multiple automated keratometry readings can also provide clues that a patient's ocular surface is not optimized. For example, in Figure 3, the IOLMaster readings show significant variability in both the magnitude of astigmatism as well as the axis of astigmatism. The three central corneal power measurements should be within 0.25 diopters in each of the principal meridians in order to be considered valid. ${ }^{12}$ Initiating therapy can help optimize the ocular surface, and result in more consistent keratometry readings. ${ }^{13}$

\section{Corneal Dystrophy}

Corneal dystrophies, specifically epithelial basement membrane dystrophy (EBMD), can be subtle and easily overlooked during routine examination. ${ }^{1}$ The consequences of missing EBMD can yield unwanted refractive results and unhappy patients. ${ }^{8}$ Similar to dry eye, EBMD can be identified by irregular astigmatism on topography. ${ }^{14,15}$ EBMD can also result in inaccurate keratometry readings. Furthermore, following refractive surgery, EBMD can reduce $B C V A .{ }^{15}$ Treatment of EBMD, which will improve BCVA, will have the unwanted side effect of altering the keratometry power. This can result in a significant shift in refractive error. ${ }^{16}$

When EBMD is identified, it is important to assess the severity of the condition. In many patients, there is limited EBMD that is not in the visual axis. In these cases, the condition can be observed and cataract surgery can be performed with a standard IOL. Sometimes it may be best to avoid ATIOLS. For example, a patient with EBMD who has significant astigmatism may benefit from a toric IOL in the short term. However, if the EBMD requires removal, the astigmatism may significantly change, or in some cases, completely resolve. As well, EBMD can result in mild loss of best corrected visual acuity (BCVA) due to irregular astigmatism, so multifocal IOLs may not be the best option as studies have shown
Figure 1: Preoperative Evaluation

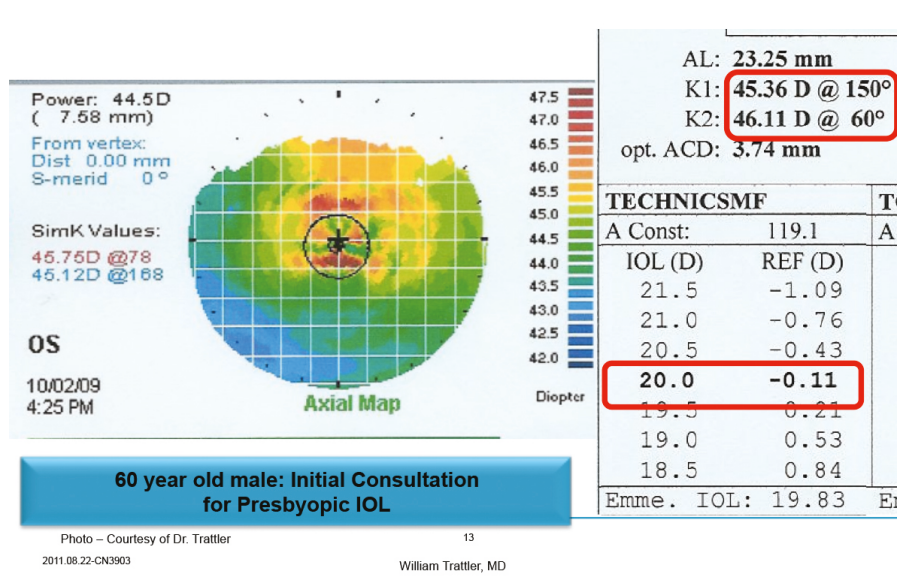

Figure 2: Dry Eye Identified

One week after cyclosporine BID \& topical steroids

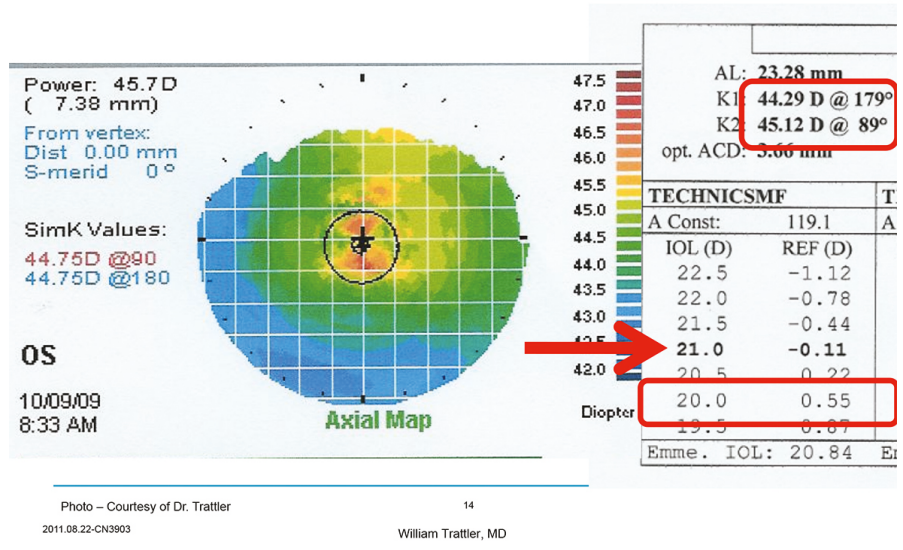

Figure 3: IOLMaster ${ }^{\circledR}$ Readings

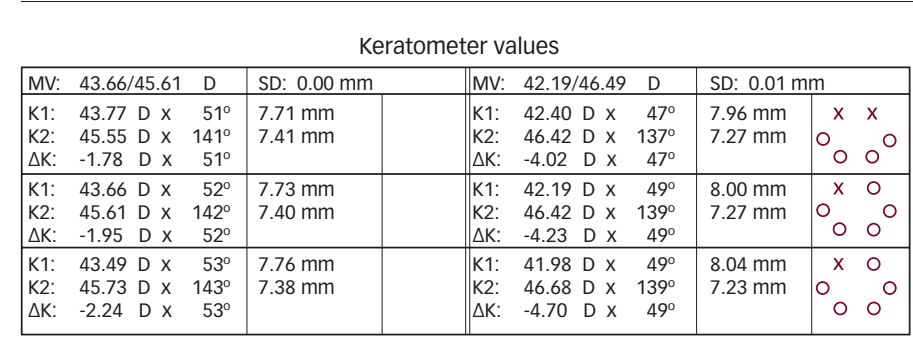

there are limitations with the use of multifocal lenses in astigmatism. ${ }^{17}$ Patients with more advanced EBMD, leading to irregular astigmatism directly in the visual axis, can benefit from treatment prior to cataract surgery. ${ }^{8}$ Identification of EBMD is made with careful evaluation at the slit lamp. Areas of epithelial irregularity and areas of negative staining will be identified (see Figure 4). Treatment of EBMD can range from simple epithelial debridement at the slit lamp to phototherapeutic keratectomy (PTK). ${ }^{18,19}$ Following these procedures, the corneal epithelium requires weeks to months to reach its final shape. ${ }^{20}$ Once the surface has stabilized, repeat biometry should be performed to select the optimal IOL power. As can be seen in Figure 5, there can be significant changes in corneal topography following treatment of EBMD. 
Figure 4: Negative Staining in Epithelial Basement Membrane Dystrophy

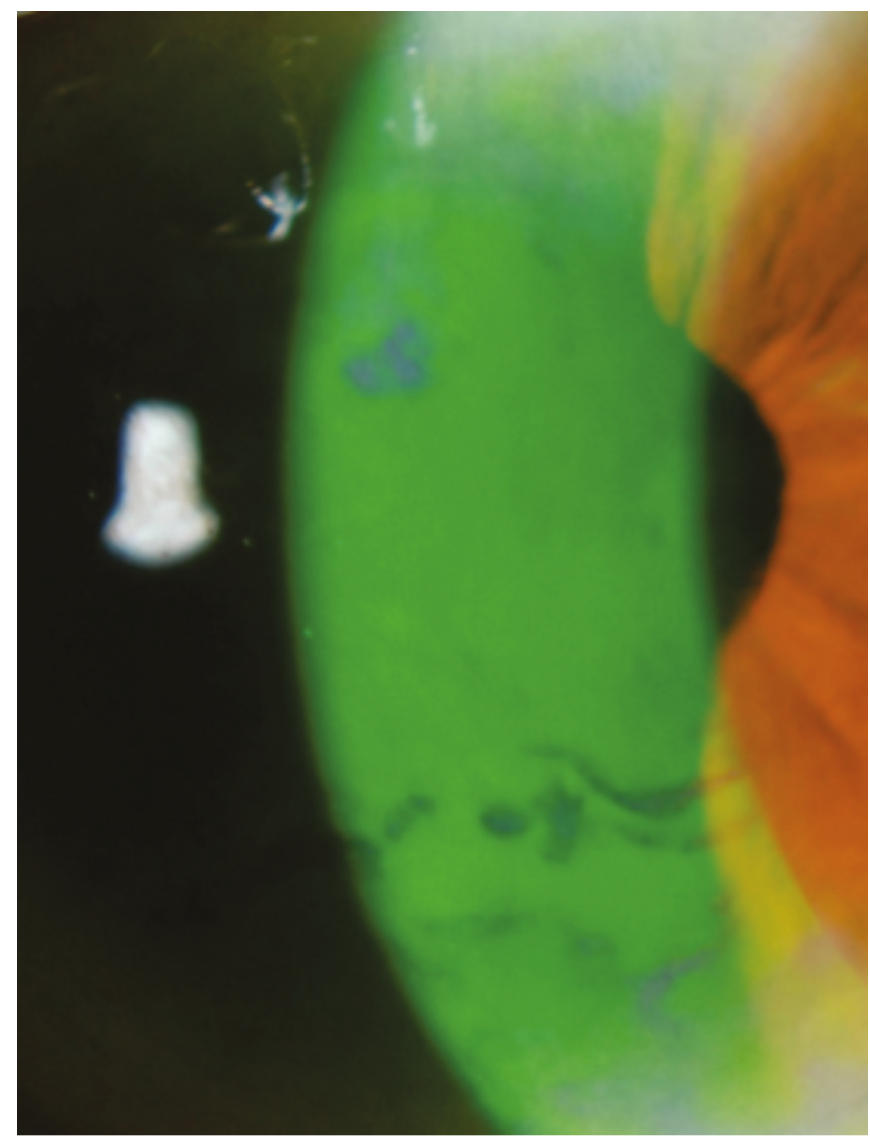

Figure 5: Epithelial Basement Membrane DystrophyManagement Options

\section{- Treat EBMD before or after cataract surgery?}

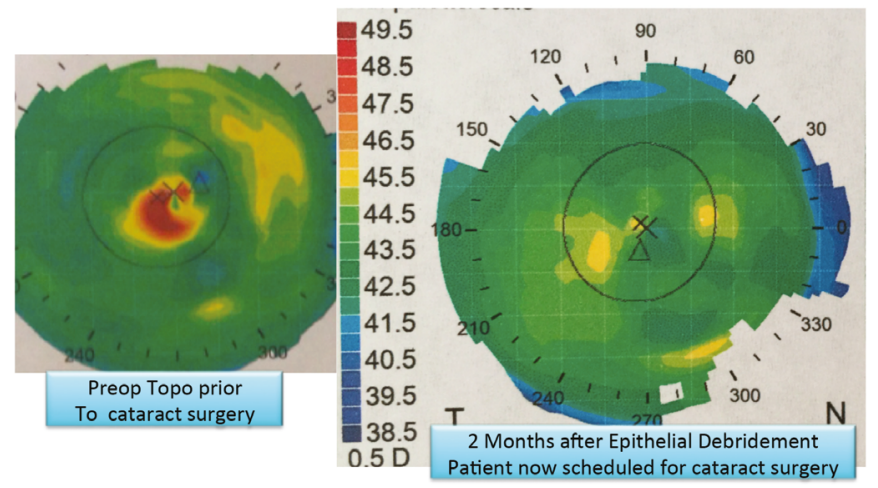

Jennifer Loh, MD

In Figure 5, the left side topography is from a patient diagnosed with EBMD on cataract surgery evaluation. The condition and treatment options were discussed with the patient, who elected to undergo superficial keratectomy. The resultant topography on the right side shows the improved corneal shape. The new keratometry measurements were used when calculating and selecting the IOL and the planned refractive outcome was achieved.
Figure 6: Using Topography to Assess Corneal Shape

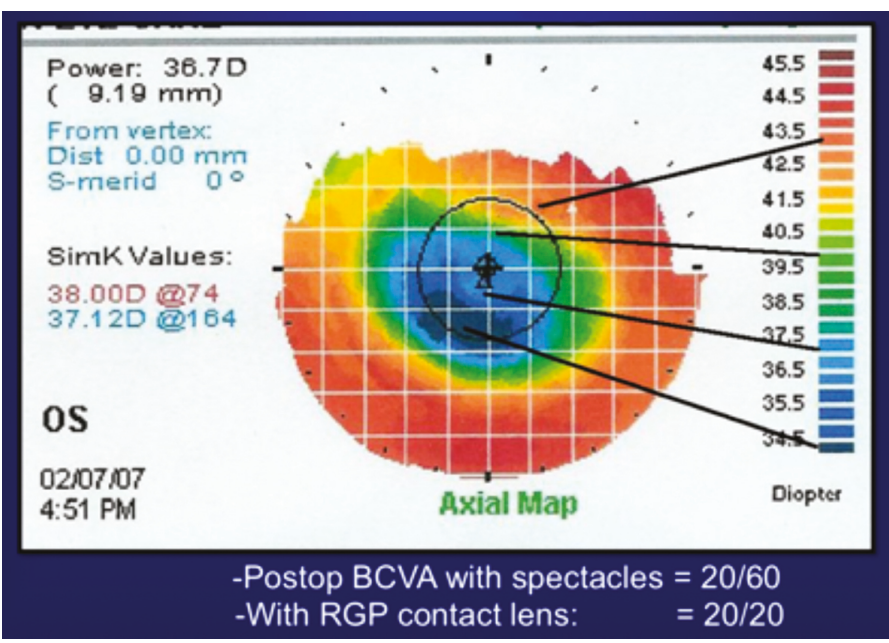

Figure 7: Identifying Pellucid Marginal Degeneration with Topography

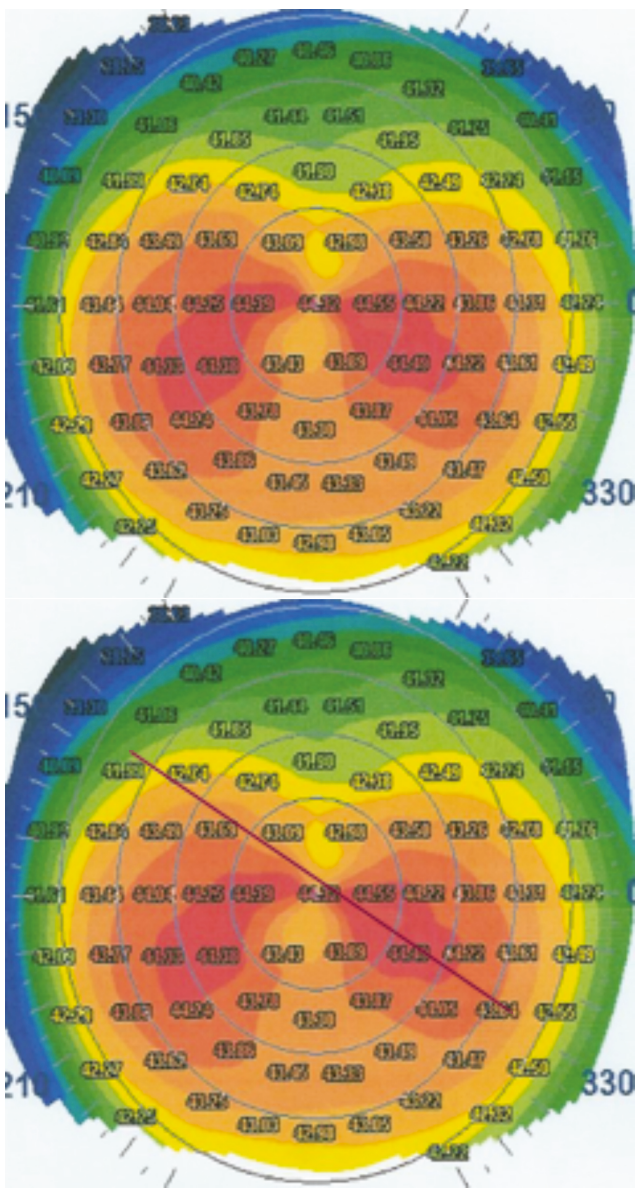

A. Topography of early pellucid marginal degeneration (PMD). B. A line drawn along one axis of the astigmatism showing how the axis should appear if orthogonal.

\section{Postrefractive Surgery}

Patients with a history of corneal laser vision correction or radial keratometry benefit from preoperative corneal topography.1., Topography determines whether the optical zone created with refractive surgery 
is centered or decentered. With myopic corneal refractive surgery, the cornea is flattened to reduce myopia. Topography reveals whether the optical zone has remained centered, or has drifted and now is not centered. A decentered optical zone can affect quality of vision.21,22 For hyperopic corneal refractive surgery, the central cornea is steepened. While the apex of the altered cornea typically remains centered, topography evaluates the corneal shape and can identify cases where the steep apex of the cornea has decentered. ${ }^{23,24}$

Preoperative topography is important in patients with previous corneal refractive surgery because it allows the cataract surgeon to effectively counsel the patient on visual expectations with cataract surgery. With corneal refractive surgery, the shape of the cornea is altered to adjust the refractive error and reduce the need for glasses. However, over time, the cornea can adjust in shape, which can affect the quality of vision. ${ }^{16}$ Topography provides important information about the shape of the cornea, and can determine whether the optical zone has symmetrical astigmatism or has a gradient of astigmatism within the visual axis. ${ }^{23,24}$ For example, this patient who underwent laser-assisted in situ keratomileusis in 1999 in Figure 6 presented for cataract surgery. Evaluation of the optical zone reveals there is a significant gradient within the visual axis. Postoperatively, despite having uncomplicated cataract surgery with a monofocal IOL, the BCVA was 20/60. However, with a rigid gas-permeable lens, the patient was corrected to 20/20.

\section{Astigmatism Management}

Toric IOLs comprise approximately $7.6 \%$ of all IOL procedures. ${ }^{25}$ Successful placement of these IOLs requires accurate measurement of the magnitude and axis of astigmatism. While manual and automated keratometers can actually be more accurate in this regard than topography, ${ }^{26}$ the data provided by topography about the corneal shape is essential to assess whether the astigmatism is truly orthogonal and to also exclude the possibility of irregular corneal astigmatism and/or corneal ectasias that would contraindicate placement of a toric IOL. ${ }^{12}$ For example, in Figure 7 the manual keratometry readings may present as $42.00 \times 44.00$. Without topography, a surgeon may place a toric IOL in the power and axis he or she believes to be correct. However, when topography is assessed, a pellucid marginal degeneration pattern appears and a skewed axis becomes apparent. With this additional information, it is evident that a toric IOL, which currently can correct only orthogonal astigmatism, may not be effective. Furthermore, over time, if the patient's corneal condition progresses, the astigmatism axis and magnitude can change. In addition, when topography identifies patients as having forme fruste keratoconus (FFKC), KCN, or pellucid, surgeons should avoid a multifocal IOL.

$\mathrm{KCN}$ is another condition that must be carefully screened for prior to cataract surgery, especially when planning for ATIOLs or any refractive corneal incisions. ${ }^{27}$ In situations where $\mathrm{KCN}$ is present, the avoidance of toric IOLS and corneal incisions is critical as refractive corneal incisions could cause worsened corneal ectasia and unpredictable results. ${ }^{28}$ Toric IOLS are generally avoided in KCN where there is no orthogonal axis of astigmatism. ${ }^{28}$ In addition, multifocal IOLs would also be contraindicated as their optical system does not function well with irregular astigmatism and any decrease in contrast sensitivity will be magnified by the corneal irregularity ${ }^{1.8}$ When $\mathrm{KCN}$ is identified in patients with cataracts, the surgeon may consider corneal cross-linking either prior to cataract surgery or following cataract surgery, as this procedure can result in improvement in corneal shape, and improvement in quality of vision..$^{29}$

\section{Summary}

Corneal topography is a powerful tool that can be employed prior to cataract surgery to assess corneal shape to help with surgical planning. Topography can help identify patients with irregular corneal shape related to ocular surface disease or EBMD that requires treatment prior to cataract surgery. Topography is also important for patients with previous corneal refractive surgery, to help determine whether a patient is an appropriate candidate for an ATIOL. Furthermore, screening with topography can identify patients with FFKC, KCN, or pellucid marginal degeneration, which allows the surgeon to properly counsel the patient on the expected visual results, as well as help with patient selection. While corneal topography is not performed preoperatively in all patients prior to cataract surgery, the valuable information provided may lead to more widespread adoption of topography as an essential planning tool in patients scheduled for surgery.
1. Hardten D, Corneal Topography and Refractive IOLS-What to Look For. In: Mastering Refractive IOLS The Art and Science, Thorofare, NJ: SLACK Inc., 2008:564-7.

2. American Academy of Ophthalmology Cataract and Anterior Segment Panel, Preferred Practice Pattern Guidelines, Cataract in the Adult Eye. San Francisco, CA: American Academy of in the Adult Eye. San Francisco, CA: American Academy of
Ophthalmology, 2011. Available at: http://www.aao.org/preferredOphthalmology, 2011. Available at: http://www.aao.org/pre (accessed September 12, 2013).

3. Trattler W, Buznego C, Corneal Topography and Refractive IOLSCase Studies. Mastering Refractive IOL The Art and Science. Thorofare, NJ: SLACK Inc., 2008:568-70.

4. Basic and Clinical Science Course Section 13: Refractive Surgery. 2008-2009, 8-10.

5. MCQueen BR, Martinez CE, Klyce SD, Corneal topography in cataract surgery, Curr Opin Ophthalmol, 1997;8:22-8.

6. Trattler W, Frank B, McCabe S, et al., Retrospective review of incidence of abnormal corneal topography in patients scheduled for cataract surgery. Presented at the American Society of Cataract and Refractive Surgery Annual Meeting, April 25, 2014, Boston, MA. Poster \#7036;

7. Trattler W, Goldberg D, Reilly C, et al., Prospective health assessment of cataract patients' ocular surface. Poster presented at: World Cornea Congress, April 8, 2010, Boston, MA.

8. Braga-Mele R, Chang D, Dewey S, et al., Multifocal intraocular Ienses: Relative indications and contraindications for implantation, J Refract Surg, 2014;40:313-22.

9. Reeves $\mathrm{S}$, Lindstrom R, Optimizing the Ocular Surface Preoperatively. Mastering Refractive $10 \mathrm{~L}$ The Art and Science.
Thorofare, NJ: SLACK Inc., 2008;571-4

10. Luchs J, Buznego C, Trattler W, Asymptomatic or minimally symptomatic blepharitis in patients having cataract surgery, ASCRS, San Diego, March 2011. Poster.

11. Caldwell M, Ashfari N, Corneal Topography-Is it Necessary? Mastering Refractive IOL The Art and Science. Thorofare, NI: SLACK Inc., 2008;557-8.

12. Hill W, Accurate Keratometry Readings. In: Cataract and Refractive Surgery Today, 2009;32-4.

13. Albietz JM, Lenton LM, Management of ocular surface and tear film, before, during, and after lasik in situ keratomileusis, J Refract Surg, 2004;20:62-71.

14. Woreta FA, Davis GW, Bower KS, LASIK and surface ablation in corneal dystrophies, Surv Ophthalmology 2015:60:115-22.

15. Rezende R, Uchoa UC, Cohen EJ, et al., Complications associated with anterior basement dystrophy after laser in situ keratomileusis, I Cataract Refract Surg, 2004;30:2328-31.

16. Rathi V, Vyas S, Sangwan V, Phototherapeutic keratectomy, Indian I Ophthalmol, 2012;60:5-14.

17. Hayashi K, Manabe S, Yoshida M, Hayashi H, Effect of astigmatism on visual acuity in eyes with a diffractive multifoca astigmatism on visual acuity in eyes with a diffractive mulitoc

18. Vo RC, Chen IL, Sanchez PJ, et al., Long term outcomes of epithelial debridement and diamond burr polishing for cornea epithelial irregularity and recurrent corneal erosion, Cornea, 2015;34:1259-65

19. Aldave AJ, Kamal K, Vo R, Yu F, Epithelial debridement and bowman's layer polishing for visually significant epithelial irregularity and recurrent erosions, Cornea, 2009:28:1085-90.
20. Rosa N, De Bernardo M, et al., Corneal aberrations before and after photorefractive keratectomy, J Optom, 2008;1:53-8.

21. Mrochen M, Kaemmerer M, Mierdel P, Seiler T, Increased higher order optical aberrations after laser refractive surgery: a problem of subclinical decentration, $J$ Cataract Refract Surg 2001:27:362-9

22. Bühren J, Yoon G, Kenner S, et al., The effect of optical decentration on lower- and higher-order aberrations after photorefractive keratectomy in a cat model, Invest Ophthalmol Vis Sci, 2007;48:5806.

23. Maeda N, Evaluation of optical quality of corneas using corneal topographers, Cornea, 2002;21(Suppl. 7):S75-8.

24. Klyce SD, Computer-assisted corneal topography. Highresolution graphic presentation and analysis of keratoscopy, Invest Ophthalmol Vis Sci, 1984;25:1426-35.

25. Market Scope's Q2-2015 Cataract Surgeon Report.

26. Chang M, Kang SY, Kim HM, Whichkeratometer is most reliable for correcting astigmatism with toric intraocular lenses, Korean 」 Ophthalmol, 2012;26:10-4.

27. Borzog S, Pineda R, Cataract and keratoconus: minimizing complications in intraocular lens calculations, Semin Ophthalmol 2014:29:376-9.

28. Visser N, Bauer NJ, Nuijts RM, Toric intraocular lenses: historical overview, patient selection, IOL calculation, surgical techniques, clinical outcomes, and complications, J Cataract Refract Surg, 2013;624-37.

29. Agrawal V, Long term results of corneal collagen crosslinking with riboflavin for keratoconus, Indian J Ophthalmol, 2013;61:433-4. 